Ilya Karzhemanov

\title{
RETRACTED ARTICLE: Some pathologies of Fano manifolds in positive characteristic
}

Received: 24 July 2014 / Accepted: 16 October 2015

Published online: 29 October 2015

The article Some pathologies of Fano manifolds in positive characteristic by Ilya Karzhemanov published under DOI:10.1007/s00229-015-0796-9 has been retracted by the journal's editorial board.

The retraction has been made because in a post-publication peer review process it was reported by three independent reviewers that the paper contains errors which invalidate its main results.

All three reviewers presented examples which show that Proposition 2.9 and Lemma 2.12 are wrong. Furthermore it was pointed out that the way the Fano variety $\mathrm{X}$ in the Main Theorem 1.4 is constructed is in contradiction with the Theorem of Campana and Kollar-Miyaoka-Mori that every smooth Fano variety over an algebraically closed field is chain connected.

The author of the paper has not admitted to the alleged errors and disagrees with the retraction.

The online version of this article contains the full text of the retracted article as electronic supplementary material.

Electronic supplementary material: The online version of this article (doi:10.1007/s00229015-0796-9) contains supplementary material, which is available to authorized users.

I. Karzhemanov ( $₫$ ): Kavli IPMU (WPI), The University of Tokyo, 5-1-5 Kashiwanoha, Kashiwa 277-8583, Japan. e-mail: ilya.karzhemanov@ipmu.jp 